Benavent Boluda, José Alfredo (alumno).

García Serrano, África (alumno).

Llamas Pacheco, Rosario (profesora).

Universidad Politécnica de Valencia,

Departamento de Conservación y Restauración de Bienes Culturales

\title{
Documentar la técnica a través de la restauración. Proceso de intervención de un ninot de falla tradicional.
}

\author{
TIPO DE TRABAJO
}

Póster.

\section{PALABRAS CLAVE}

Restauración; fallas; técnica ejecución.

KEY WORDS

Restoration; "fallas"; artistic technique.

\section{RESUMEN}

El trabajo presenta el interesante proceso de restauración llevado a cabo en un "ninot" de falla del año 1961. El artista fallero Julián Puche Ferrándiz trabajó durante cinco años para la comisión de la falla Na Jordana, periodo en el cual realizó este boceto que posteriormente se convertiría en falla. Nuestro objeto presenta, desde el momento de su creación, una naturaleza específica: no se trata de un "ninot indultat", ya que nunca fue concebido para arder, sino de un estudio previo que posteriormente quedó guardado y expuesto en el casal fallero. Está pues, dotado de cierta singularidad, pues presenta elementos añadidos con respecto a lo que sería un clásico "ninot de falla indultat". A su vez, la calidad de la obra es sobresaliente, por lo que nos encontramos ante una creación que atesora los valores que la hacen merecedora de conservación.

Por otro lado, el trabajo realiza un profundo estudio de la técnica de ejecución tradicional, de manera que queda caracterizada la factura de nuestro objeto, proceso imprescindible antes de poder emprender la restauración. De este modo, han quedado recogidos y documentados los aspectos que se refieren al plano material de la obra, pero también al plano conceptual, para que, una vez conocida la intención artística, el estado de conservación de la materia, y las posibles discrepancias entre ellos, pudieran tomarse las decisiones adecuadas de conservación.

También hemos realizado una detallada descripción del estado de conservación y el proceso de restauración llevado a cabo, con el fin de que pueda ser conocido por otros restauradores, ya que la intervención sobre este tipo de patrimonio no es demasiado conocida y por lo tanto, nuestra aportación puede tener un carácter útil realmente. Por último se han realizado algunas recomendaciones sobre la conservación preventiva a adoptar, dada la necesidad de almacenar o exhibir el objeto correctamente.

\section{ABSTRACT}

The paper presents the interesting process of restoration carried out in a "ninot de falla" from 1961. The fallas artist Julian Puche Ferrándiz worked for five years for the commission of the Na Jordana, a period in which he made this sketch later would become monument. Our object has, since its creation, a specific nature: there isn't a "indultat ninot" because it was never meant to burn, but a previous study that was subsequently saved and displayed in the at the headquarters. It is endowed with certain uniqueness, and has added elements relative to what would be a classic "ninot indultat." In turn, the quality of work is outstanding, so we have a creation that holds the values that make it worthy of preservation. 
On the other hand, the work makes a thorough study of traditional execution, so that is characterized invoice our object, essential process before they can undertake restoration. Thus, they have been collected and documented aspects relating to the material of the work, but also the conceptual plane, so that, once known artistic intention, the state of conservation of matter, and possible discrepancies between them, may take appropriate conservation decisions.

We have also made a detailed description of the state of conservation and restoration process carried out, in order that it may be known by other restaurateurs, since the intervention on this heritage is not widely known and therefore, our contribution can have a really useful character. Finally there have been some recommendations to adopt preventive conservation, given the need to store or display the object correctly.

\section{CONTENIDO}

Esta obra representa el remate central del monumento de la comisión Falla Na Jordana de Valencia del año 1961. El "ninot", está formado por un busto erguido de un hombre sobre una peana de madera que sostiene a su vez un cohete y un buñuelo, símbolos de la fiesta valenciana. Bautizado por el artista como so Quelo, es conocido comúnmente como El llaurador. Fue modelado por el artista con la técnica tradicional para que expresara dos emociones diferentes según el día de las fiestas falleras en que se encontrara. En la tarde del día 15 de Marzo, anterior al día de la "plantà", y con el juego de luces y sombras que se crea en el rostro con la luz natural, el "ninot" parece llorar por haber sido separado del resto de "ninots" con los ha permanecido durante todo el año. Sin embargo, durante la "nit de la plantà", en la que se monta toda la escenografía de los "ninots", so Quelo es iluminado de manera que muestre una carcajada de alegría como muestra de orgullo por formar parte del monumento.

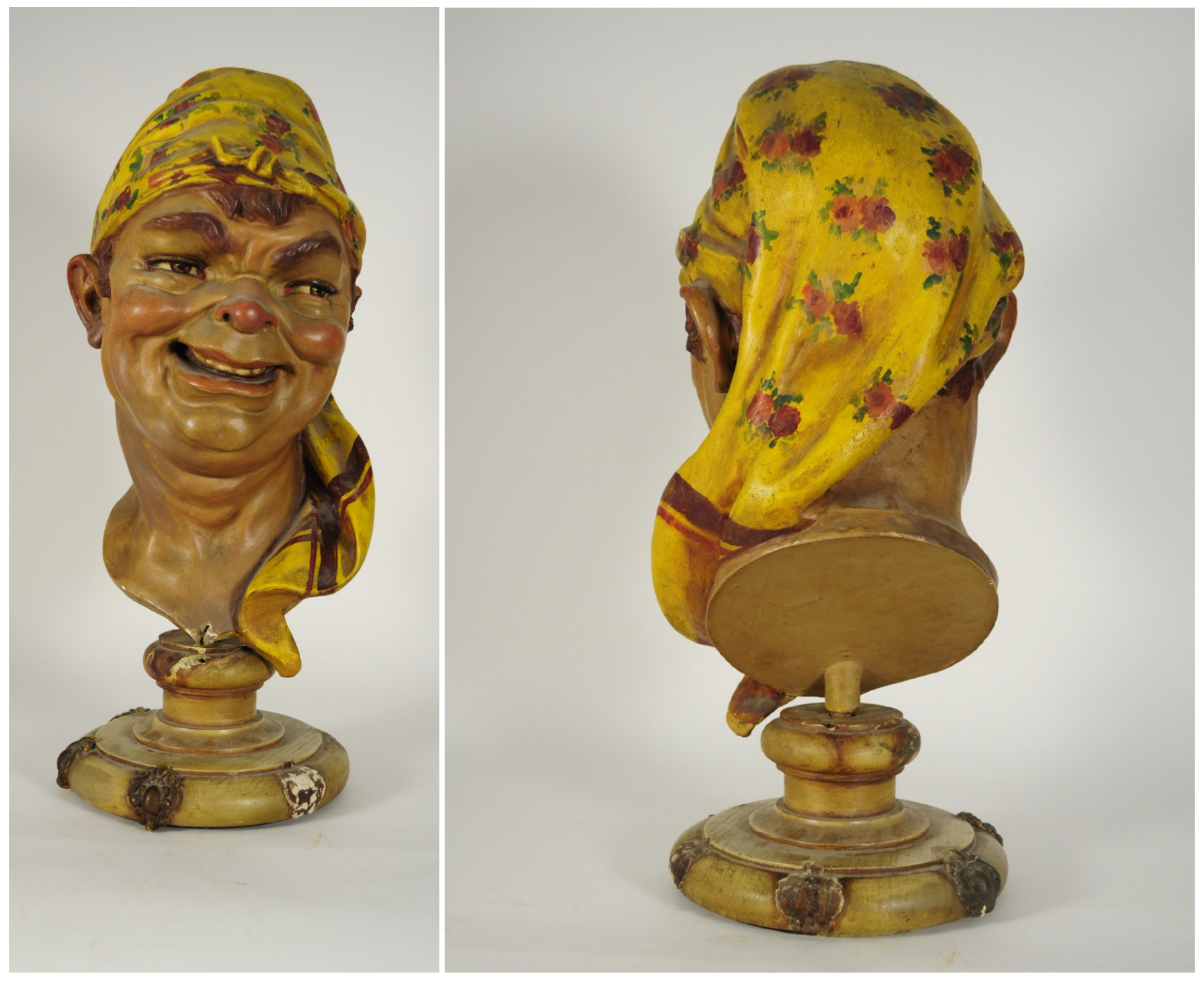

Ilustración 1 Fotografías generales de la obra con luz visible. Anverso y reverso. 


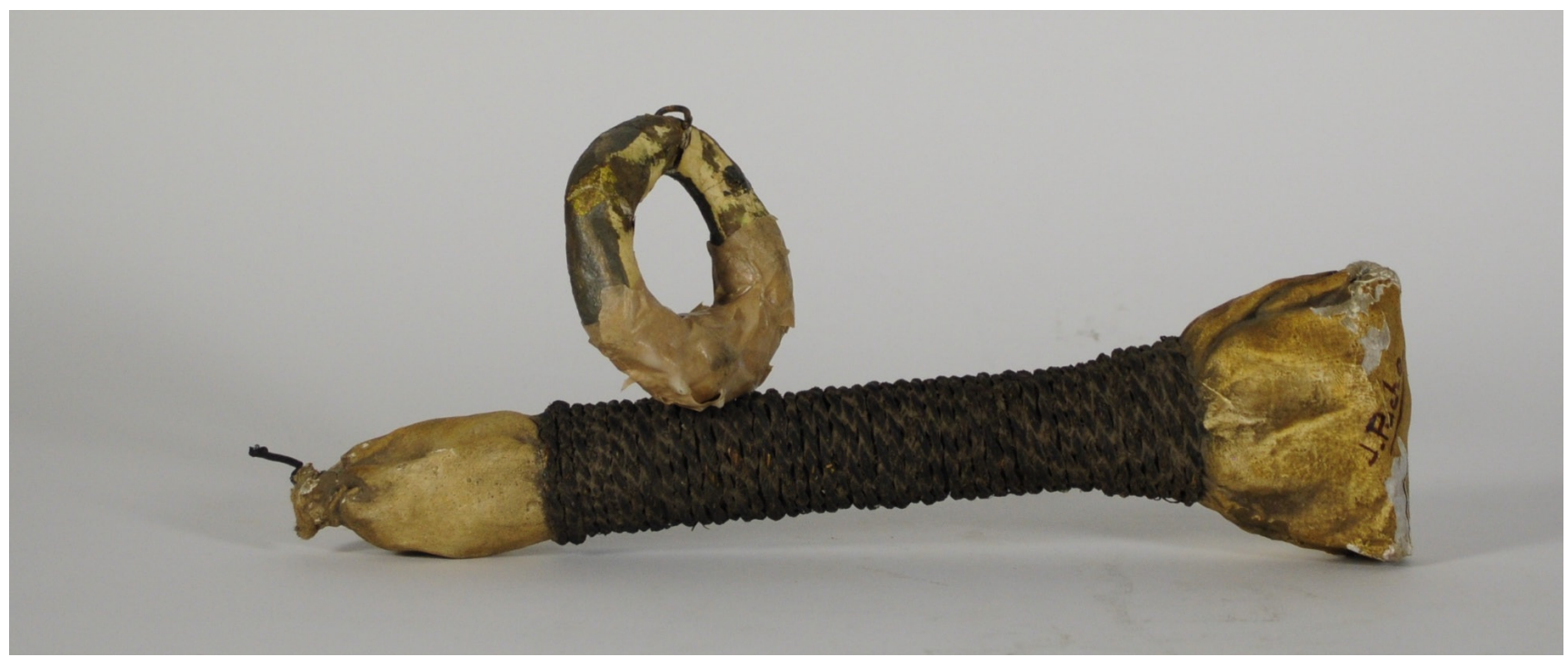

Ilustración 2 Fotografía generale del cohete y el buñuelo que también forman parte de la obra.

La obra intervenida es un boceto que realizó el artista para la comisión. La ubicación en la que se encontraba no permitía un fácil acceso, ya que se almacenaba en un estante decorativo a una altura aproximada de dos metros. La pieza no estaba protegida por ninguna vitrina ni por ningún material de preservación, por lo que presentaba abundante suciedad superficial de manera generalizada.

\section{Estado de conservación}

El estado de conservación de la obra no era bueno. Presentaba diversos tipos de patologías, como una importante pérdida de soporte, preparación, y película pictórica, en la parte delantera del cuello, producida probablemente por efecto de un golpe. La naturaleza del catón piedra, de carácter altamente higroscópico, y el paso del tiempo, han convertido al material base de la obra en rígido y quebradizo, y por lo tanto resulta muy vulnerable a los impactos.

Otra importante patología presente era una grieta en la zona de unión del busto al soporte de madera que lo sujeta, grieta que incluye pérdida de preparación y película pictórica.

Por otro lado, encontramos abundante presencia de repintes, sobre todo en la parte inferior del busto, así como pequeñas lagunas y arañazos distribuidos por toda la superficie. Las piezas que se encontraban en peor estado de conservación eran el buñuelo y el cohete, ambos con graves pérdidas, repintes y problemas de unión al busto. 


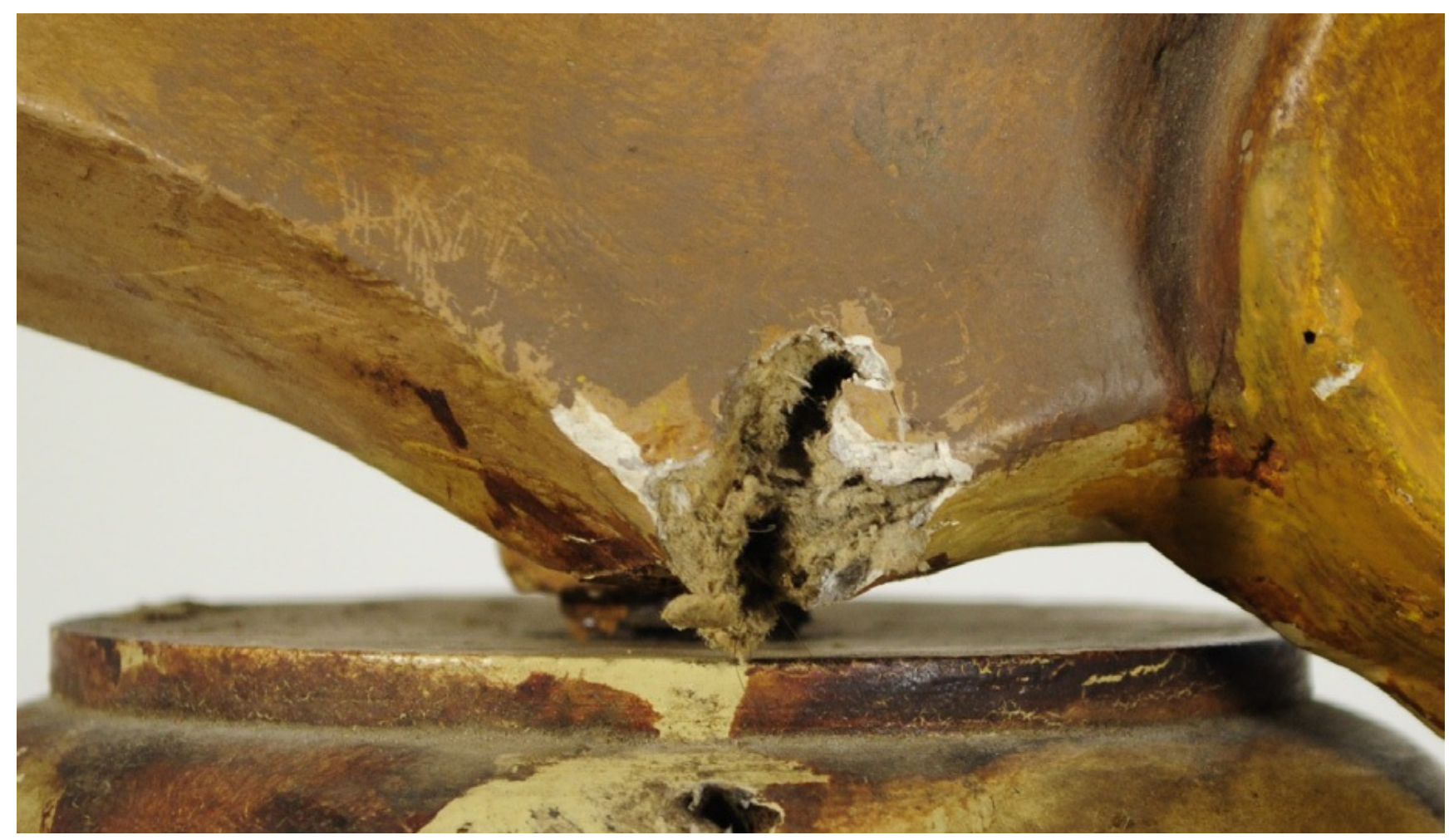

llustración 3 Pérdida de soporte, preparación y película pictórica. En esta imagen se puede observar el grosor de las diversas capas.

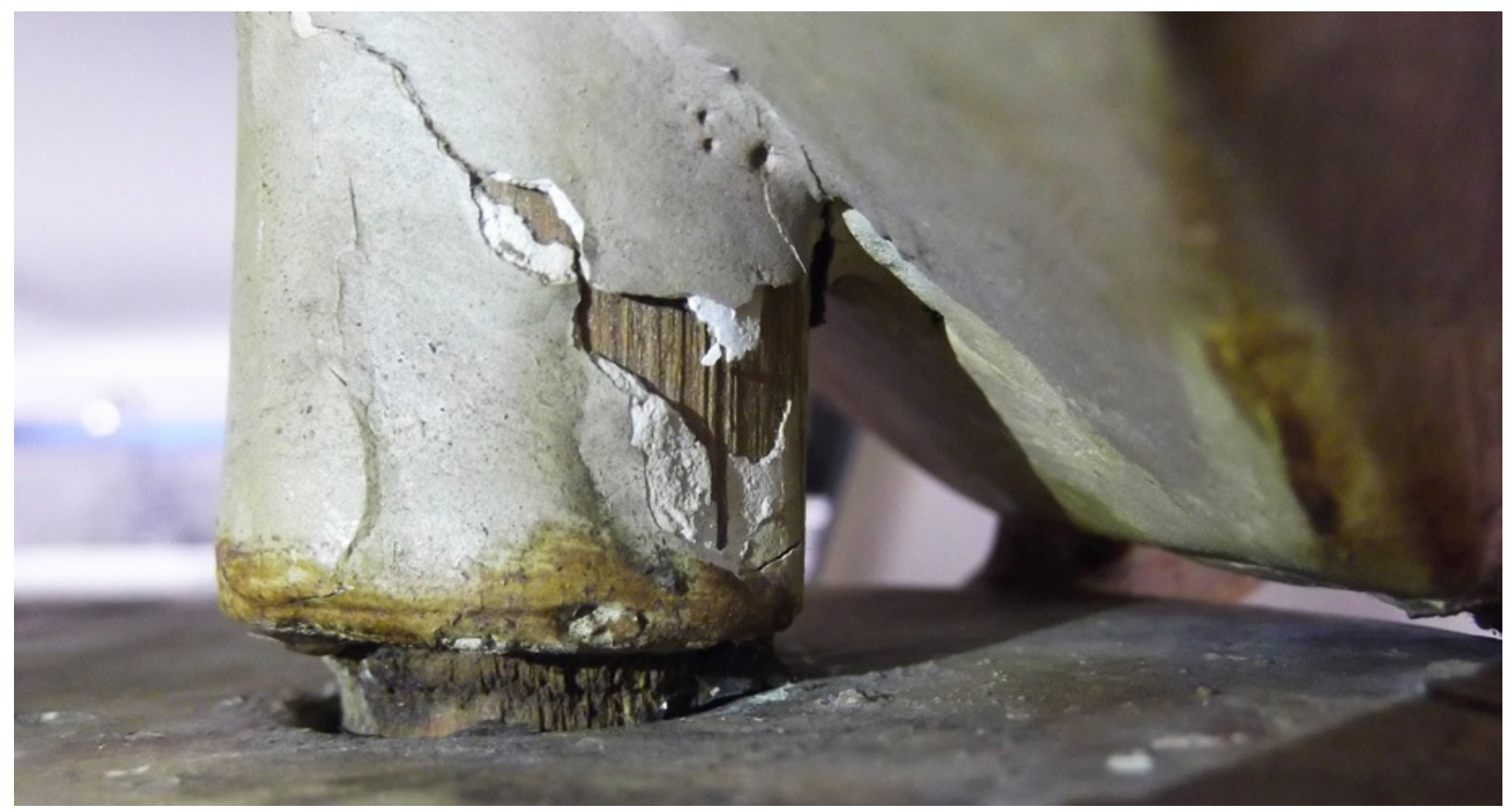

llustración 4 Detalle de la grieta junto con la pérdida de preparación y película pictórica, dejando a la vista el soporte de madera. 

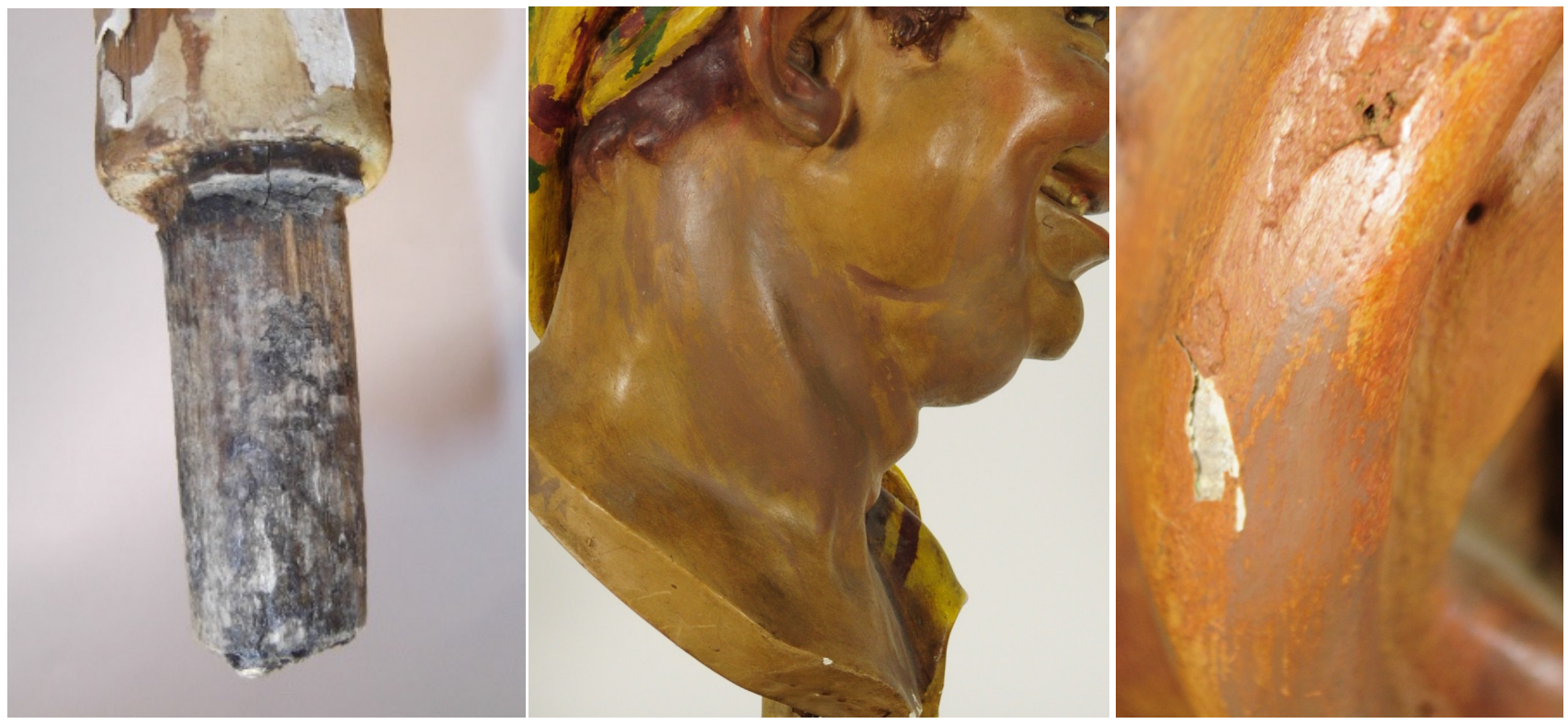

llustración 5 Detalle del estado de conservación del elemento de unión entre el busto y la peana. Repintes en la zona del cuello, oreja y barbilla. Pérdida de película pictórica y parte de imprimación en la zona de la oreja.

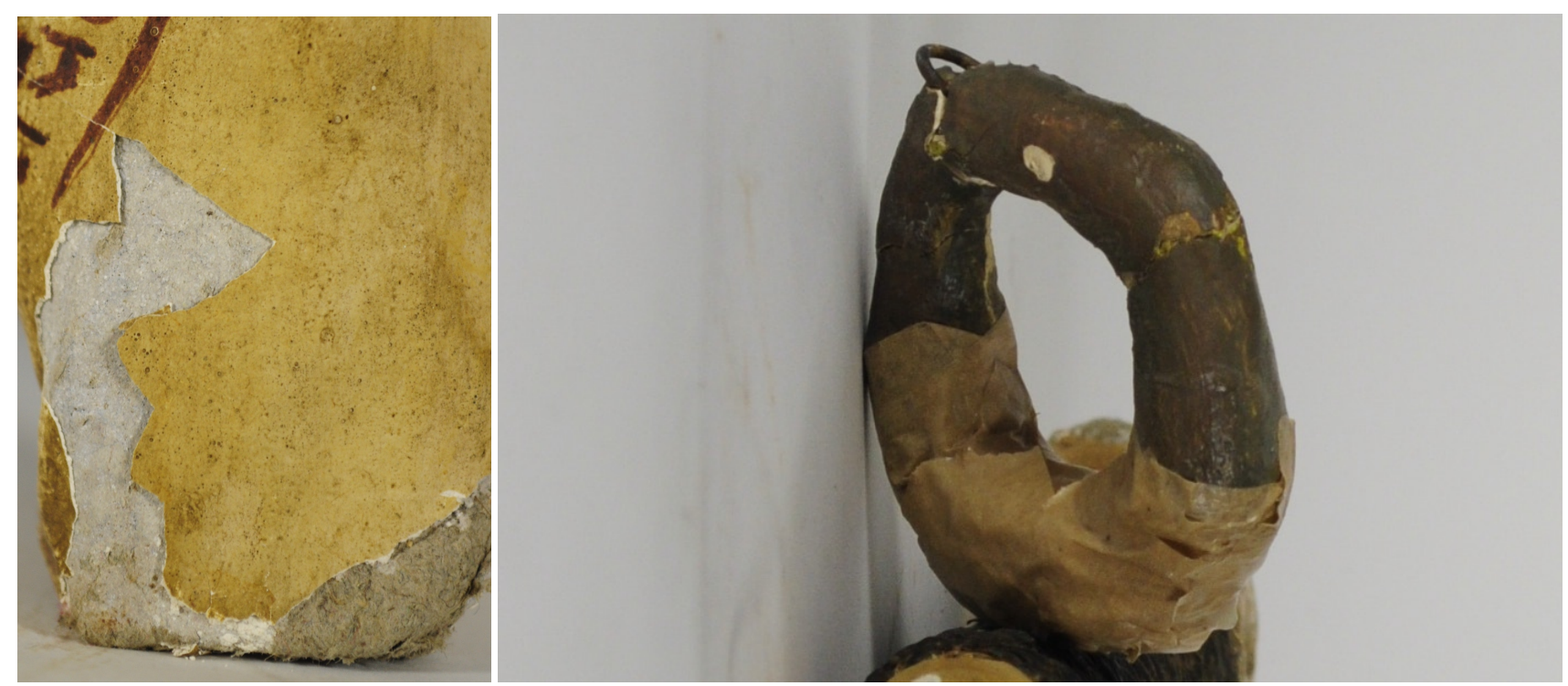

Ilustración 6 Detalle de la pérdida de preparación y película pictórica en la parte trasera del cohete. Detalle de la cinta o papel encolado en la parte inferior del cohete.

\section{Proceso de intervención.}

El objetivo del presente trabajo fue devolver a la pieza su completa lectura mediante procesos de conservación y restauración que respetasen al máximo los criterios de mínima intervención, respeto al original y reversibilidad. El proceso de intervención comenzó con la consolidación de las capas pictóricas con una sustancia filmógena de origen natural y tipo acuoso como es la cola de pescado. Para la remoción de la gruesa capa de suciedad se utilizó una brocha suave junto con la aspiración controlada. La limpieza continuó con la limpieza acuosa y para ello se empleó un tensoactivo de carácter no iónico diluido al $4 \%$ en agua desionizada. Se optó por un tratamiento acuoso, ya que las pruebas de solubilidad de los disolventes efectuadas anteriormente, dieron en el caso del agua óptimos resultados de limpieza. No obstante, se decidió introducir un tensoactivo neutro para mejorar y facilitar la hidrosolubilización de la suciedad de mayor incrustación. 
Tras la eliminación de repintes por medio químico, se procedió a realizar la reconstrucción volumétrica de los faltantes que alteraban la correcta lectura de la pieza. Para ello se preparó una pasta de cartón en polvo mezclado en una solución de $4 \mathrm{~g}$ de cola de pescado en $100 \mathrm{ml}$ de agua. Después de su secado, se aplicaron mediante pincel fino las capas de imprimación.

Por último, se impermeabilizaron los estucos y una vez secos, se reintegraron con colores al agua. Sobre la reintegración cromática se aplicó una capa de barniz satinado, consiguiendo un mejor ajuste del color, así como la protección de la capa pictórica.

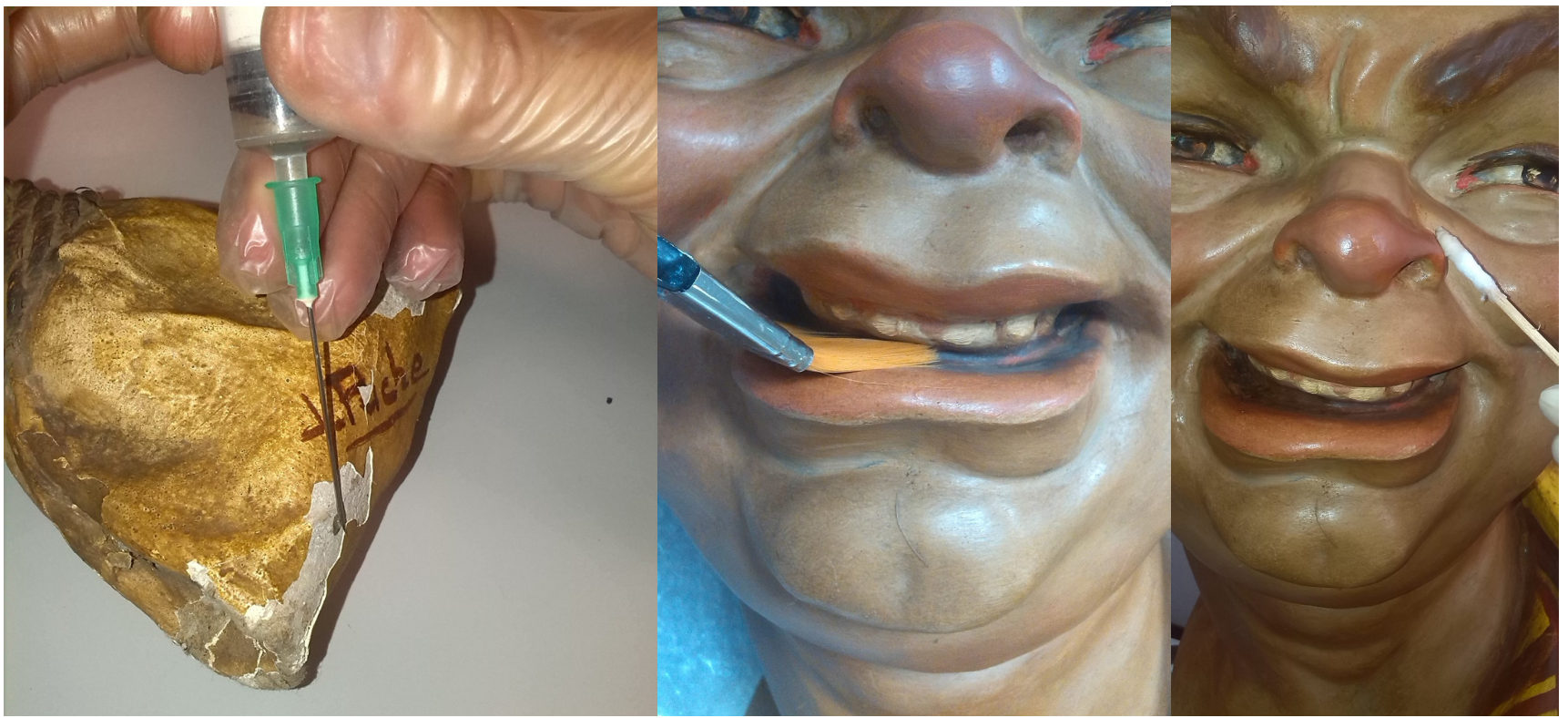

llustración 7 Inyección del consolidante. Eliminación de la suciedad superficial mediante brocha. Durante la limpieza con un tensoactivo no iónico.
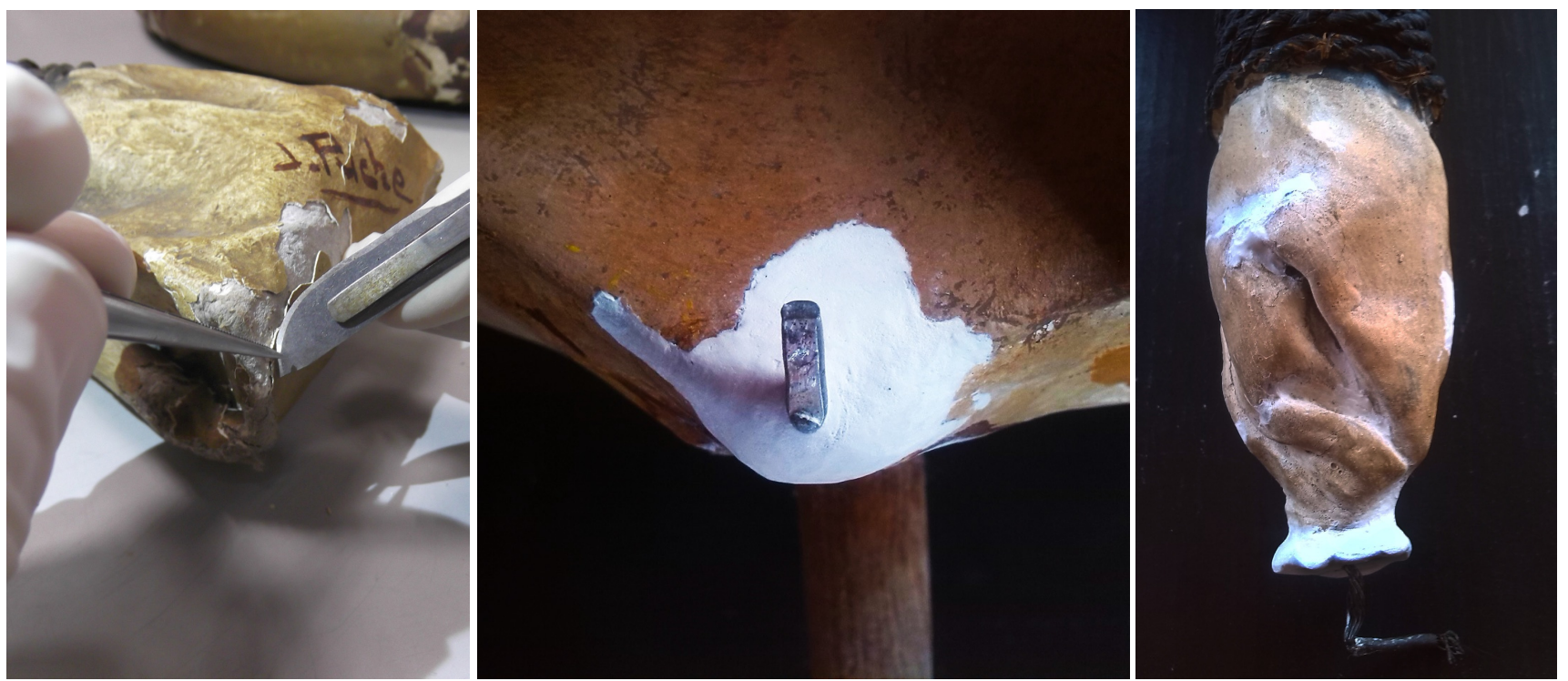

\section{Ilustración 2 Durante el proceso de consolidación y estucado.}

En conclusión podríamos decir que la intervención se ha centrado en devolver la estabilidad estructural al conjunto y en realizar una correcta limpieza que incluyera tanto los restos depositados como los repintes realizados a lo largo del tiempo. 


\section{FUENTES REFERENCIALES.}

PAStOR, J., BORREgo, P; MAROtO, J.V; BUtrAgUeÑo, J.: Llibre Noces d'Argent Falla Plaça Na Jordana (1950, 1975), Ed. Falla Plaça Na Jordana, València, 1975.

CENNINI, C., El libro del arte, Ed. Akal, Madrid, 1988.

COLOMINA, A., La preservació dels vestigis de l'art efímer de les falles. Matèria, técnica i estètica. Estudi constitutiu i anàlisi estructural, Tesis doctoral, Ed. Universitat Politécnica de València, València, 2006.

LLAMAS, R., Arte contemporáneo y restauración. Cómo investigar entre lo material, lo esencial y lo simbólico, Ed. Tecnos, Madrid, 2014. 